\title{
Behavioural Study of Concrete Surface Crack Filling Conditions on Surface Rayleigh Wave
}

\author{
Chi Hoe Liew ${ }^{1}$, Foo Wei Lee ${ }^{1}$, Yee Ling Lee ${ }^{1}$ and Ming Han Lim $^{1}$ \\ ${ }^{1}$ Lee Kong Chian Faculty of Engineering and Science, Universiti Tunku Abdul Rahman, Jalan Sungai Long, Bandar Sungai Long, \\ Cheras, 43000 Kajang, Selangor, Malaysia
}

\begin{abstract}
In the recent studies of concrete surface crack non-destructive test (NDT), the environmental factors are seldom to be taken into assessment consideration using Rayleigh wave (R-wave) propagation method. Dust, fine sand or water that accumulated in the concrete surface cracks might potentially affect the behaviour of Rwave when it propagated through the cracks. In this study, the effects of filling conditions in concrete surface crack to the properties of R-wave were examined. The targeted parameters that used for studying the behaviour of R-wave are the arrival time of R-wave and its corresponding amplitude. Numerical simulations were conducted for determining the changes of R-wave amplitudes and arrival time when it propagated through a concrete model with a surface crack which containing various filling conditions. Sand and charcoal powder were the materials used for filling the concrete cracks. The results of numerical simulations were then justified by experimental measurements. The comparison between the results of numerical simulation and experimental measurement generated a conclusion where R-wave arrival time and its corresponding amplitude is independent from the predefined filling conditions of concrete surface crack.
\end{abstract}

\section{Introduction}

These surface opening cracks often can be found from civil structures. Causes of these cracks can be caused by many different natures: physical, chemicals, mechanical or due to concrete shrinkage during setting [1]. These cracks expose the internal of concrete to the open environment. It generally interconnects flow paths and increases the permeability of concrete [2]. This eventually allows penetration of water or other agents that potentially reduce the durability of concrete [3]. From an economic point of view, repairing a defected building structure is usually more beneficial than building a new one. However, need not be repaired will be more favorable [1]. For this purpose, inspection is required for the defected concrete structures to assess their integrity and functionality in order to determine the level of necessity for remedial work to be conducted. NDT is then come into practice to perform crack assessment.

Elastic wave method is among a portion of mainstream NDT techniques applied for identifying defects and damages of concrete structures [4]. Recently, the elastic surface wave has been studied broadly because of its extensive variety of uses in acoustics, seismology, telecommunications, geophysics and material science to give some examples [5].

The special characteristic of R-waves grants it permission to be applied in various activities involving health monitoring of concrete structure. Approximately two-thirds of the total transmitted energy is believed to be carried by R-waves [6,7] and the attenuation induced by geometrical spreading is low when compared to body waves [3,8]. In addition, even in highly dispersive heterogeneous medium, concrete, for example, these low attenuation and high energy content make it much easier for the generation and record of R-wave. Researches have been performed on a diversity of application of R-waves, for example, using R-waves to characterise surface breaking crack through numerical model and determination of crack in concrete structures with an efficient procedure by the use of spectral ratios between the incident and transmitted R-waves [9]. Despite this, NDT methods adopting the principle of propagation of Rwave for crack assessment seldom consider the environmental factors that could possibly affect the behaviour of R-wave. Droplets of rain or air contaminant are the materials that could stagnate in the concrete surface cracks and potentially affect the R-wave behaviour when it propagated through the cracks. Hevin et al. [9] suggested that future perspectives are to broaden the method to the case of more realistic cracks (waterfilled cracks, cracks with mechanical contact between the two lips and etc.). This indicates the importance and necessity of conducting a behavioural study of surface Rwave propagated through the concrete medium with different surface crack filling conditions, in order to 
optimize and realize in situ inspection with the R-wave method.

This study focuses on two types of filling materials: fine charcoal powder and fine sand and water and aimed to investigate the effect of the proposed filling materials to the propagation of R-wave. The charcoal powder was used because of its major component is black carbon, which is similar to soot, one of the pollutants, which is mainly emitted from motor vehicles. It is also originated from incomplete fuel combustion; either diesel or gasoline [10]. Normal saltation event of sand will bring sand particles into the surface opening cracks of concrete structures.

\subsection{Numerical Simulation}

Multi-channel R-wave assessment method was deployed in this study. A series of numerical simulations were conducted and the results are then examined to gain further insight into the R-wave interaction with a crack filled with different type of material. Using a commercially available software, Wave 2000 by CyberLogic Incorporation, the motions of elastic waves were simulated. The function of it is to solve the twodimensional (2D) acoustic (elastic) wave equation based on a method of finite difference and simulating received waveforms under a variety of spatial and temporal acoustic interrogations, give as Equation (1) [11]:

$\rho \frac{\partial^{2} w}{\partial^{2} t}=\left[\mu+\eta \frac{\partial}{\partial t}\right] \nabla^{2}+\left[\lambda+\mu+\phi \frac{\partial}{\partial t}+\frac{\eta}{3} \frac{\partial}{\partial t}\right] \nabla(\nabla \cdot w)(1)$

where $\rho$ is material density, $\lambda$ is the first Lame constant, $\mu$ is the second Lame constant, $\eta$ is shear viscosity, $\phi$ is bulk viscosity, $\nabla$ is the gradient of operator, $\nabla \cdot$ is the divergence operator, $\partial$ is the partial differential operator, $t$ is the time and $w$ is a two dimensional column vector whose components are the $x$ and $y$ components of the displacement of the medium at location $(x, y)$.

The properties of the concrete and the filling materials were listed in Table 1 , with the material and geometry of the concrete medium were set as uniform throughout the simulation work. In addition, Table 2 shows the parameters of the investigation, including the frequency of excitation, type of filling materials and volume of crack to be filled. Figure 1 depicts the filled cracked simulation model with a dimension of $500 \mathrm{~mm}$ (width) x $100 \mathrm{~mm}$ (depth) in size. Four sensors linear array was adopted in this study, where two of the sensors were located before and after the crack. Infinite boundary conditions were configured at the left, right and bottom sides of the model to ensure that transmitted waves would not be reflected when it reached the edge of the model.

Table 1. Simulation Materials Properties

\begin{tabular}{|c|c|c|c|}
\hline Parameter & Concrete & Sand & Charcoal \\
\hline $\begin{array}{c}\text { First Lame constant, } \\
\lambda(\mathrm{MPa})\end{array}$ & 11100 & 0.482 & 1.381 \\
\hline
\end{tabular}

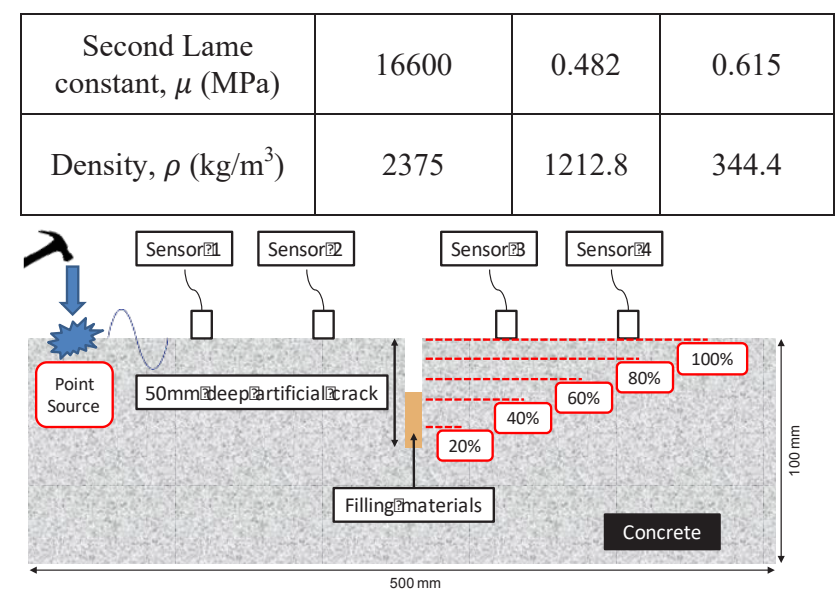

Fig. 1. Schematic Diagram of Concrete Simulation Model Introduced by a Vertical Surface Crack with Filling Material (Side View)

Table 2. Characteristic of Simulated Crack

\begin{tabular}{|c|c|c|}
\hline $\begin{array}{c}\text { Type of } \\
\text { materials }\end{array}$ & $\begin{array}{c}\text { Volume of crack to be } \\
\text { filled (\%) }\end{array}$ & $\begin{array}{c}\text { Frequency of } \\
\text { source excitation, } \boldsymbol{f} \\
(\mathbf{k H z})\end{array}$ \\
\hline $\begin{array}{c}\text { Charcoal } \\
\text { powder }\end{array}$ & $20,40,60,80$ and 100 & $\begin{array}{c}20,40,60,80 \text { and } \\
100\end{array}$ \\
\hline $\begin{array}{c}\text { Fine } \\
\text { Sand }\end{array}$ & $20,40,60,80$ and 100 & $\begin{array}{c}20,40,60,80 \text { and } \\
100\end{array}$ \\
\hline
\end{tabular}

\subsection{Experimental Measurement}

The concrete specimen was cast with a water-to-cement ratio of 0.51 , density of $2375 \mathrm{~kg} / \mathrm{m}^{3}$, modulus elasticity of $25 \mathrm{GPa}$ and maximum aggregate size of $20 \mathrm{~mm}$. The artificial surface crack was created by placing a polystyrene board with a width of $5 \mathrm{~mm}$ at the middle of the concrete specimen with a depth of $50 \mathrm{~mm}$. The concrete mix properties are given in Table 3.

Table 3. Properties of Concrete Specimen

\begin{tabular}{|c|c|c|c|}
\hline $\begin{array}{l}\text { Cement } \\
\left(\mathrm{kg} / \mathrm{m}^{3}\right)\end{array}$ & $\begin{array}{c}\text { Water } \\
\left(\mathrm{kg} / \mathrm{m}^{3}\right)\end{array}$ & $\begin{array}{c}\text { Fine } \\
\text { aggregate } \\
\left(\mathrm{kg} / \mathrm{m}^{3}\right)\end{array}$ & $\begin{array}{c}\text { Coarse } \\
\text { aggregate } \\
\left(\mathrm{kg} / \mathrm{m}^{3}\right) \\
\end{array}$ \\
\hline 314.0 & 160.0 & 702.1 & 130.4 \\
\hline
\end{tabular}

This study adopts a four-sensor array measurement as shown in Figure 2. This configuration is defined as a linear measurement array. The steel ball impacts were implemented by the same operators so that the inconsistencies in the generation of stress waves can be minimized. Changing the size of steel ball impactors made it possible for generation of R-waves with varying dominant frequencies. The sizes of the steel balls used in this study were $1.0 \mathrm{~cm}, 1.5 \mathrm{~cm}, 2.0 \mathrm{~cm}$ and $2.5 \mathrm{~cm}$. It is attempted to investigate the relations between dominant frequency, amplitude and velocity of R-wave when propagated through the surface crack filled with different materials and different volume. It should be noted that a thin layer of petrol gel couplant was applied between the sensors and the specimen surface so that a good 
acoustical coupling is ensured. For each measurement, ten excitations were conducted from both ends of the measurement array. The recorded waveforms were then stacked accordingly to enhance signal persistency for further processing.

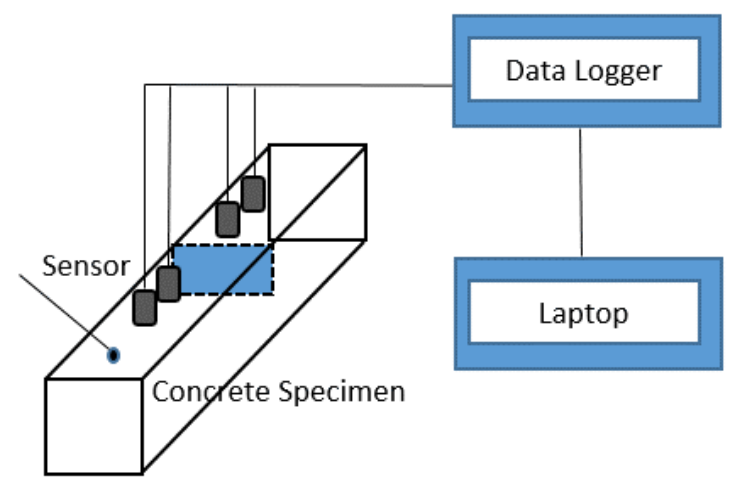

Fig. 2. Laboratory Measurement Setup: Schematic Representations of Sensor Arrangement

\section{Results}

It is reasonable to assume that, the filled crack will only affect the properties of the simulated waveforms that recorded by sensor $3(\mathrm{~S} 3)$ and sensor $4(\mathrm{~S} 4)$. Therefore, the waveforms that obtained from S3 and S4 will mainly be discussed, in order to study the behaviour of R-waves after propagated through a crack that containing a different type of filling.

\subsection{Simulation Result: Sand and Charcoal Powder-Filled Crack}

Figures 3 and 4 show the typical waveforms obtained from S3 for the plain and sand-filled as well as charcoal powder-filled cases, respectively. As can be seen from the figures, the acquired waveforms remain almost the same and show identical characteristics in terms of amplitude and arrival time of R-wave component regardless of the percentage of filling materials. The accumulated filling materials may cause a bridging effect and increase the possibility of the elastic wave to be directly propagated through the crack. However, as shown in the simulation results, the arrival time and amplitude of R-wave is not affected by the presence of filling materials. This can be proved by the insignificant percentage difference in R-wave arrival time (less than $1.0 \%$ ) and amplitude (less than $1.8 \%$ ) as compared to the plain cracked model.

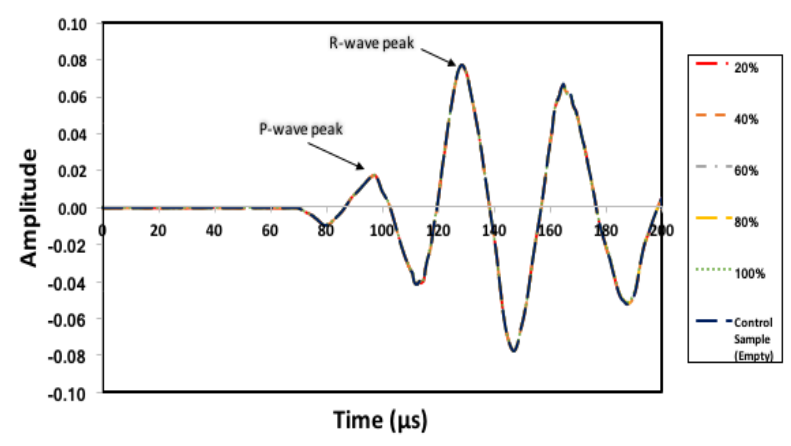

(a)

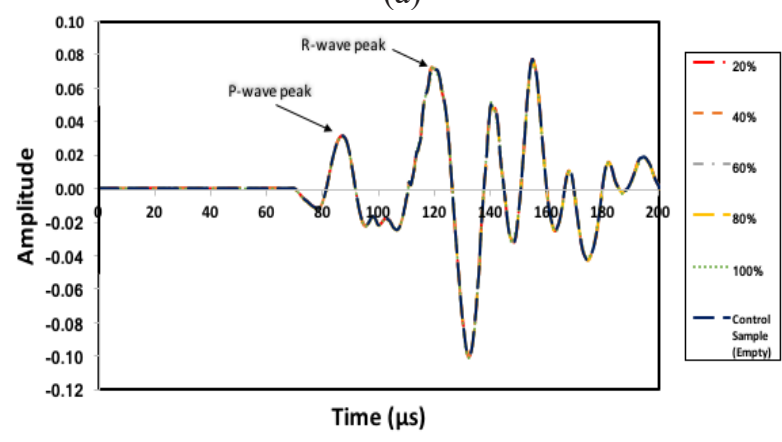

(b)

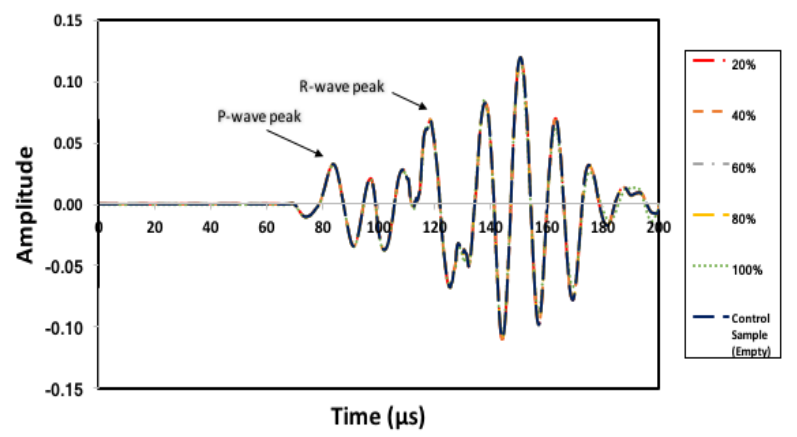

(c)

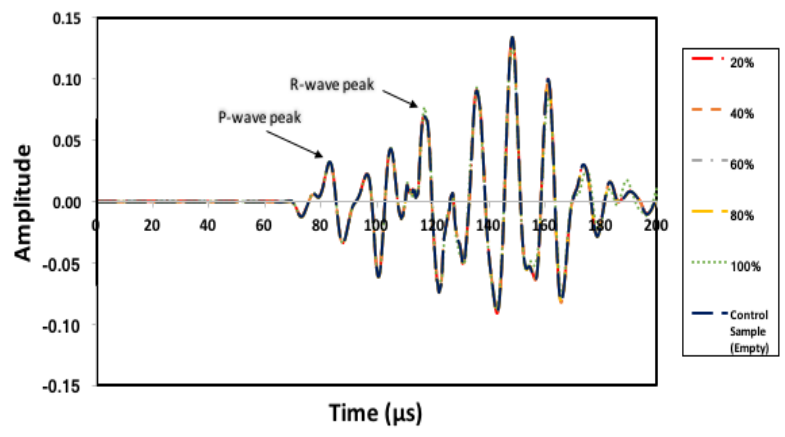

(d) 


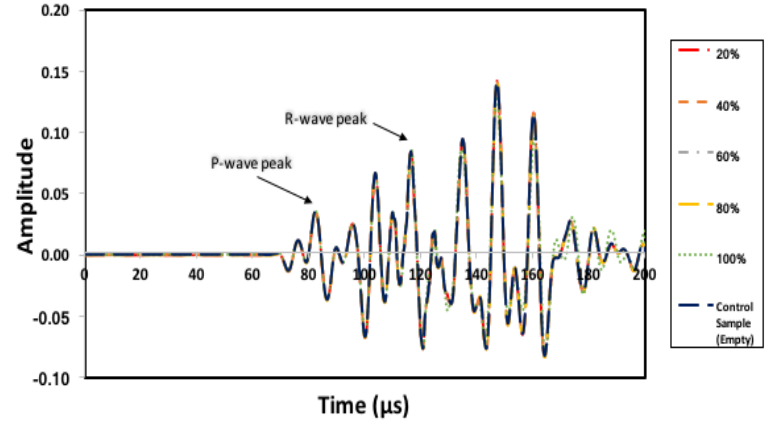

(e)

Fig. 3. Comparison of Simulated Waveforms Collected of S3 (sensor after the crack) with Different Amount of Fillings for Plain Cracked Model and (a) $20 \mathrm{kHz}$ Sand-Filled, (b) $40 \mathrm{kHz}$ Sand-Filled, (c) $60 \mathrm{kHz}$ Sand-Filled, (d) $80 \mathrm{kHz}$ Sand-Filled and (e) $100 \mathrm{kHz}$ Sand-Filled models

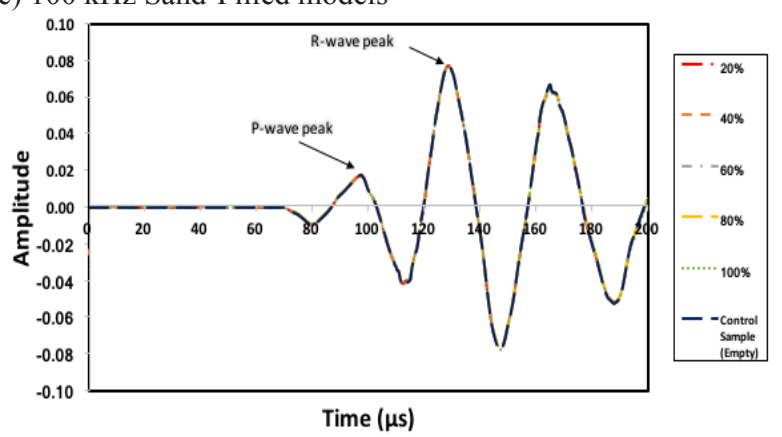

(a)

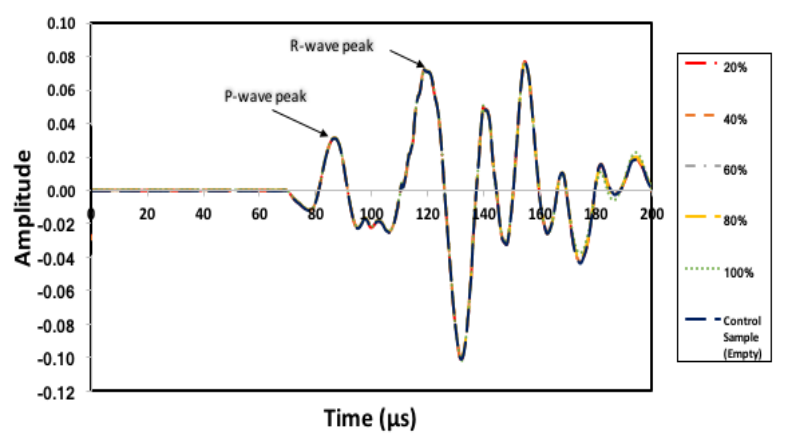

(b)

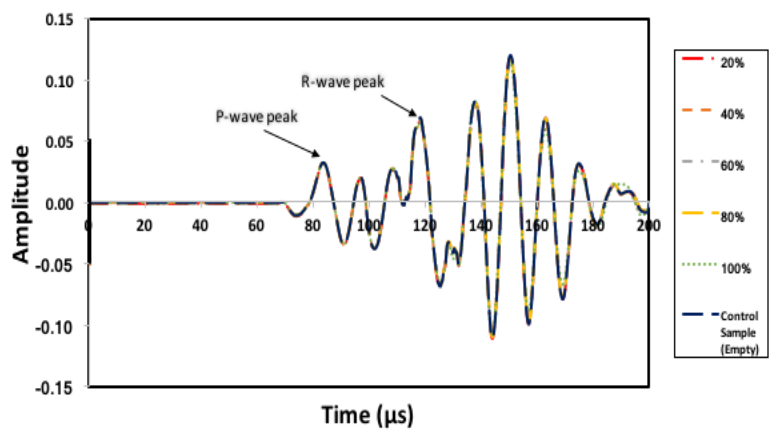

(c)

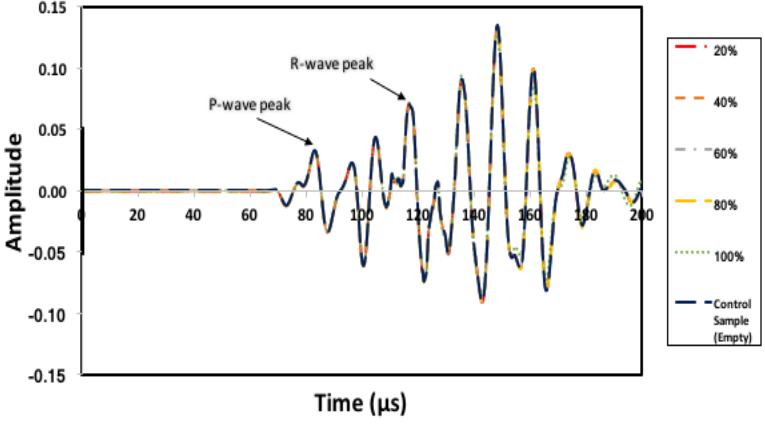

(d)

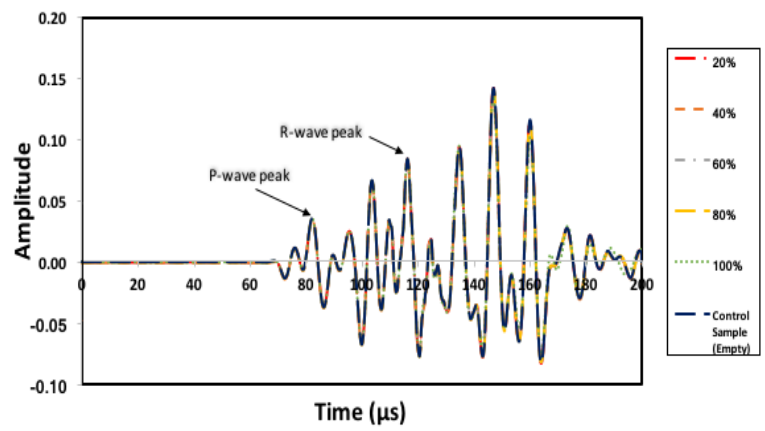

(e)

Fig. 4. Comparison of Simulated Waveforms Collected of S3 (sensor after the crack) with Different Amount of Fillings for Plain Cracked Model and (a) $20 \mathrm{kHz}$ Charcoal Powder-Filled, (b) $40 \mathrm{kHz}$ Charcoal Powder-Filled, (c) $60 \mathrm{kHz}$ Charcoal Powder-Filled, (d) $80 \mathrm{kHz}$ Charcoal Powder-Filled and (e) 100 $\mathrm{kHz}$ Charcoal Powder-Filled models

\subsection{Experimental verification}

Figures 5 and 6 show the experimental waveforms collected by S3 (sensor after the crack) for sand-filled and charcoal powder-fill cases, respectively. The experimental results justified the findings obtained from the simulation that the arrival time of $\mathrm{R}$-wave is insensitive to the filling conditions. An average of $3.0 \%$ is recorded as compared both of the filled cases to the plain cracked cases. Contrary, an opposite phenomenon was observed when analysing the amplitude of the Rwave. It can be seen that the $\mathrm{R}$-wave amplitude is more likely to be influenced by the filling condition of the surface crack, as much as $37.03 \%$ and $31.15 \%$ of difference were calculated for the sand-filled and charcoal powder-filled cases, respectively. Also, the excitation frequency and percentage of fillings again exhibit an insensitivity towards the propagation behaviour of R-wave. Similar findings as reported in the previous section. The high percentage of difference between the experimental measurement and numerical simulation is most likely due to the $2 \mathrm{D}$ numerical simulation of elastic wave, as the actual measurement involves a more complicated 3D behavior of $\mathrm{R}$-wave propagation. In addition, the sides and bottom boundaries of simulation model were simulated as infinity boundaries, which is not perfectly showing the actual situation where the reflection of the wave from the boundaries is anticipated and causing a significant 
difference between the simulation and experimental measurement.

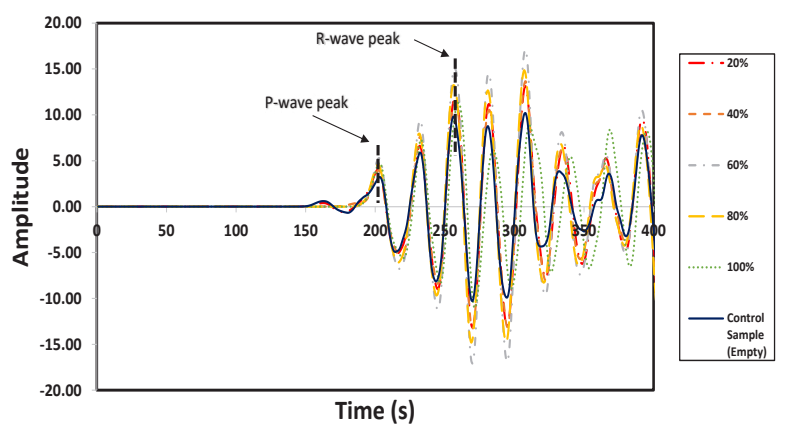

(a)

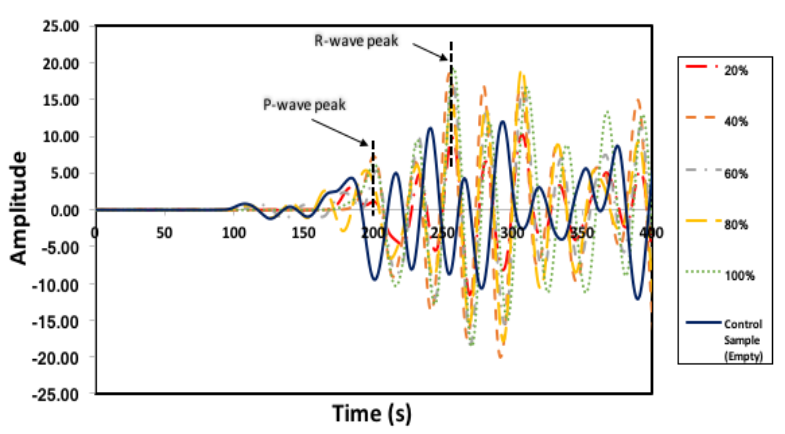

(b)

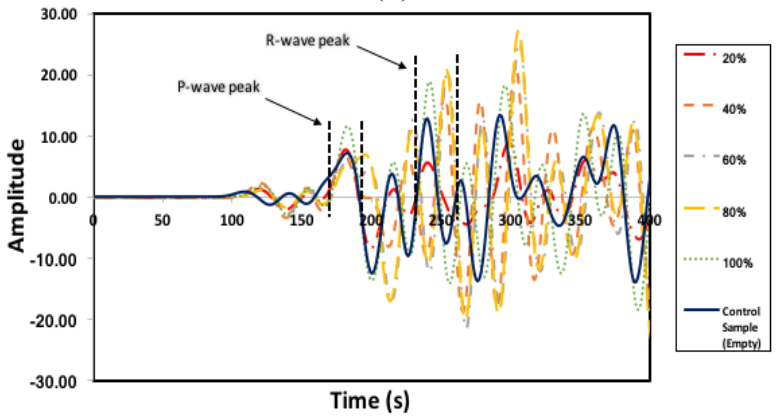

(c)

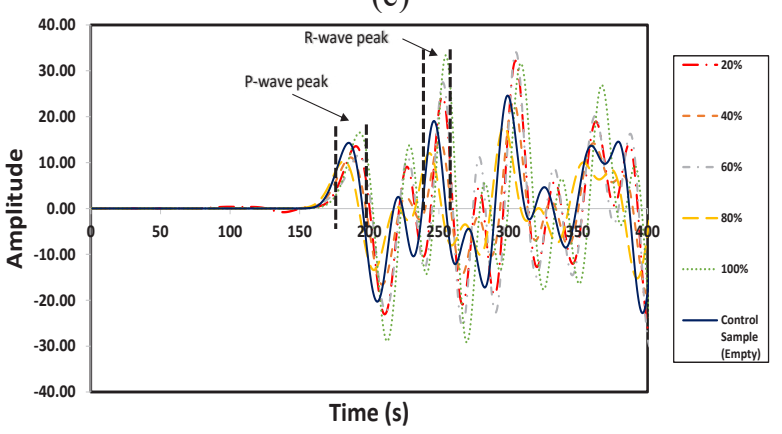

(d)

Fig. 5. Comparison between Experimental Waveforms Collected by S3 (sensor after the crack) Propagated through Plain Cracked Model and Sand-Filled Crack with Various Amount of Fillings: Excitation Done by (a) $1.0 \mathrm{~cm}$, (b) $1.5 \mathrm{~cm}$, (c) $2.0 \mathrm{~cm}$ and (b) $2.5 \mathrm{~cm}$ Diameter Steel Ball.

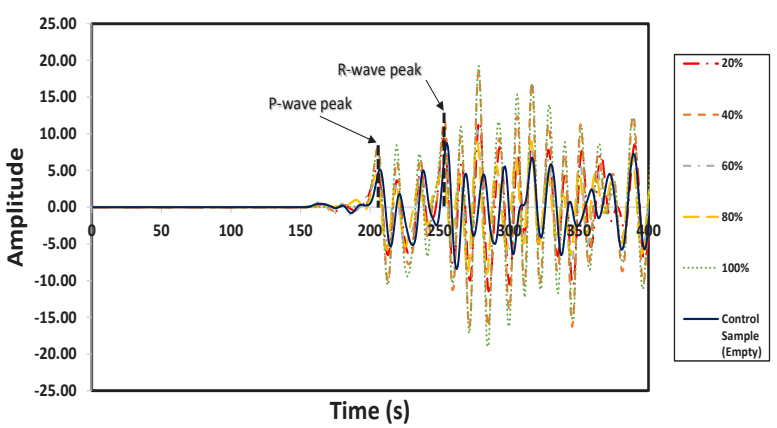

(a)

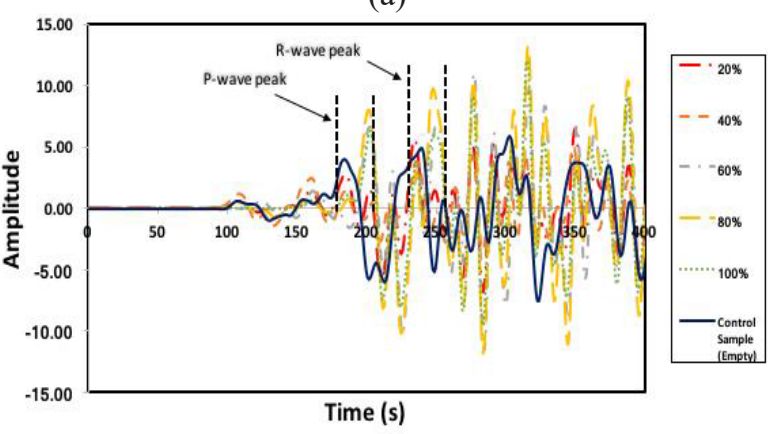

(b)

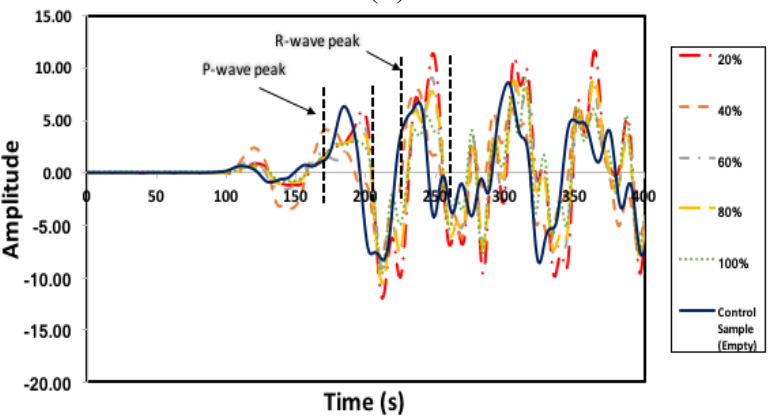

(c)

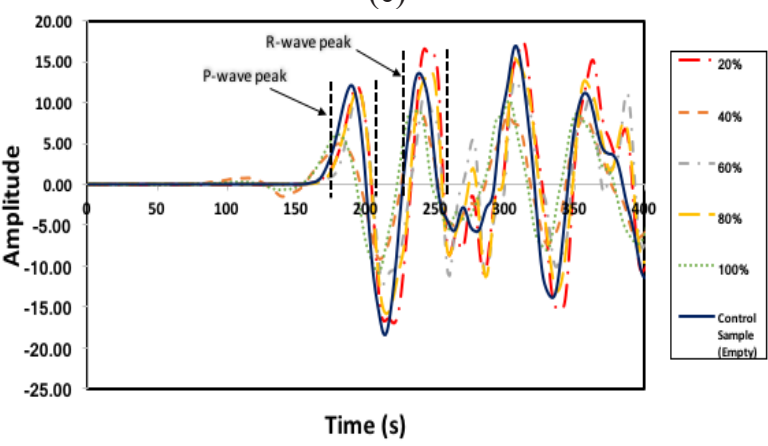

(d)

Fig. 6. Comparison between Experimental Waveforms Collected by S3 (sensor after the crack) Propagated through Plain Cracked Model and Charcoal Powder-Filled Crack with Various Amount of Fillings: Excitation Done by (a) $1.0 \mathrm{~cm}$, (b) $1.5 \mathrm{~cm}$, (c) $2.0 \mathrm{~cm}$ and (b) $2.5 \mathrm{~cm}$ Diameter Steel Ball

\section{Conclusion}

Based on the results obtained from the numerical simulations and experimental measurement, it can be concluded that the arrival time of R-wave is independent regardless the filling materials and the volume. The 
difference of R-wave arrival time is less than $1 \%$ in all the simulation cases and averaged around $3 \%$ in all the experimental measurements.

R-wave amplitude is sensitive to both the sandfilled and charcoal powder-filled experimental cases. However, there is no traceable trend can be determined in these changes. The massive differences in experimental R-wave amplitude could be possibly due to several reasons, e.g. unavoidable inconsistent impacting strength in generating R-wave on the concrete specimen, the poor acoustic coupling between the sensors and surface of the concrete specimen.

\section{Acknowledgments}

This work was supported under the University Research Funding (IPSR/RMC/UTARRF/2017-C2/L02).

\section{References}

1. Shah, A.A. and Ribakov, Y., 2008. Non-destructive measurements of crack assessment and defect detection in concrete structures. Materials \& Design, 29(1), pp.61-69.

2. Wang, K., Jansen, D.C., Shah, S.P. and Karr, A.F., 1997. Permeability study of cracked concrete. Cement and concrete research,27(3), pp.381-393.

3. Aggelis, D. G. and Shiotani, T., 2007. Repair evaluation of concrete cracks using surface and through-transmission wave measurements. Cement and Concrete Composites, 29(9), pp. 700-711.
4. Lee, F.W., Chai, H.K. and Lim, K.S., 2017. Characterizing concrete surface notch using Rayleigh wave phase velocity and wavelet parametric analyses. Construction and Building Materials, 136, pp.627-642.

5. Sudheer, G., Lakshmi, M.H. and Rao, Y.V., 2017. A note on formulas for the Rayleigh wave speed in elastic solids. Ultrasonics, 73, pp.82-87.

6. Park, C.B., Miller, R.D. and Xia, J., 1999. Multichannel analysis of surface waves. Geophysics, 64(3), pp.800-808.

7. Sung, W.S., 2008. Elastic Rayleigh Wave for Nondestructive Health Monitoring of Concrete Structure.

8. Chai, H.K., Momoki, S., Kobayashi, Y., Aggelis, D.G. and Shiotani, T., 2011. Tomographic reconstruction for concrete using attenuation of ultrasound. Ndt \& E International, 44(2), pp.206-215.

9. Hevin, G., Abraham, O., Pedersen, H.A. and Campillo, M., 1998. Characterization of surface cracks with Rayleigh waves: a numerical model. NDT \& E International, 31(4), pp.289-297.

10. Taib, M.M., Alimin, A.J., Amirnordin, S.H. and Abd Rahman, H., 2009. Reduction of soot emission from diesel fuelled engine using a novel after treatment system. Proceedings of MUCEET2009 Malaysian Technical Universities Conference on Engineering and Technology, pp. 1-6.

11. Wave2000, Cyber-Logic, Inc., New York, http://www.cyberlogic.org. 